\title{
MICROSCOPIC DERIVATION OF THE ONE-QUBIT KRAUS OPERATORS FOR AMPLITUDE AND PHASE DAMPING
}

\author{
M. Arsenijević ${ }^{1}$ and N. Banković ${ }^{2}$ \\ ${ }^{1}$ Department of Physics, Faculty of Science, Kragujevac, Serbia \\ e-mail: momirarsenijevic@kg.ac.rs \\ ${ }^{2}$ Technical College of Applied Studies in Kragujevac, Kragujevac, Serbia
}

(Received March 8, 2016)

\begin{abstract}
This article presents microscopic derivation of the Kraus operators for (the generalized) amplitude and phase damping process. Derivation is based on the recently developed method [Andersson et al, J. Mod.Opt. 54, 1695 (2007)] which concerns finite dimensional systems (e.g. qubit). The form of these operators is usually estimated without insight into the microscopic details of the dynamics. The behaviour of the qubit dynamics is simulated and depicted via Bloch sphere change.
\end{abstract}

\section{INTRODUCTION}

Quantum information processing substantially depends on the mathematical details of the environmental influence exerted on the qubit-registers [1]. Nevertheless, to the best of our knowledge, theoretical origin of the widely used Kraus operators for the one-qubit quantum noise-channels [1-7] has not been investigated yet. In this paper we perform a thorough analysis of the microscopic models for the standard one-qubit amplitude damping and phase damping quantum processes. To this end we use a recently formulated method [8] for derivation of the Kraus operators from a microscopic master-equation description of the processes. We find the unitary equivalence of the here derived Kraus operators with those widely used in the literature, thus presenting the same quantum noise process. The converse conclusion regarding the one-qubit depolarizing process will be presented elsewhere [9].

In Section 2, we overview the method of Andersson et al [8]. In Section 3 we derive 
the Kraus operators for the generalized amplitude damping process, while in Section 4 we derive the Kraus operators for the phase damping process. Section 5 is the conclusion.

\section{OVERVIEW OF THE METHOD}

In the paper [8], the authors developed a general procedure for deriving a Kraus decomposition from the known master equation and vice versa, regarding the finite-dimensional quantum systems. The only assumption is that the master equation is local in time.

The so-called Nakajima-Zwanzig projection method [11,13] gives the following master equation for the system's density operator $\hat{\rho}_{S}(t),(\hbar=1)$ :

$$
\frac{d \hat{\rho}_{S}(t)}{d t}=-i\left[\hat{H}, \hat{\rho}_{S}(t)\right]+\int_{0}^{t} \mathcal{K}_{t, s}\left[\hat{\rho}_{S}(s)\right] d s
$$

where $\hat{H}$ represents the system's self-Hamiltonian (that includes the so-called Lamb-shift term) and $\mathcal{K}_{t, s}$ is the memory kernel which accounts for the non-unitary effects due to the environment.

Certain processes can be written in a local-in-time form $[11,13]$ :

$$
\dot{\hat{\rho}}_{S}(t)=\Lambda_{t}\left(\hat{\rho}_{S}(t)\right)
$$

where $\Lambda_{t}$ is a linear map which preserves hermiticity, positivity and unit trace of $\hat{\rho}_{S}(t)$ and has the property:

$$
\operatorname{tr} \Lambda_{t}\left(\hat{\rho}_{S}(t)\right)=0
$$

Alternatively, dynamics can be presented in a non-differential, "integral" form $[8,11$, $13]$ :

$$
\hat{\rho}_{S}(t)=\phi_{t}\left(\hat{\rho}_{S}(0)\right)
$$

where $\phi_{t}$ is a completely positive and trace preserving linear map.

It can be shown [8] that linear maps $\Lambda_{t}$ and $\phi_{t}$ are connected via the matrix differential equation:

$$
\dot{F}=L F
$$

where the matrix elements of $L$ are given by:

$$
L_{k l}=\operatorname{tr}\left[\mathcal{G}_{k} \Lambda\left(\mathcal{G}_{l}\right)\right]
$$

In eq.(6), $\left\{\mathcal{G}_{k}\right\}$ is any orthonormal basis of the Hermitian operators acting on the system's Hilbert space. For the time independent $\Lambda_{t}$, i.e. $L$, eq.(5) has the unique solution:

$$
F=e^{L t}
$$


Complete positivity of the map $\phi_{t}$ (and hence of the matrix $F$ ) is equivalent to the positivity of the, so called, Choi matrix, $S[8,10]$, whose elements are defined as [8]:

$$
S_{n m}=\sum_{s, r} F_{s r} \operatorname{tr}\left[\mathcal{G}_{r} \mathcal{G}_{n}^{\dagger} \mathcal{G}_{s} \mathcal{G}_{m}\right]
$$

With the use of equation (8), eq.(4) takes the form:

$$
\phi\left(\hat{\rho}_{S}(0)\right)=\sum_{n m} S_{n m} \mathcal{G}_{n} \hat{\rho}_{S}(0) \mathcal{G}_{m}^{\dagger}
$$

which, after diagonalization of the $S$ matrix:

$$
S=U D U^{\dagger}
$$

gives rise to a Kraus decomposition. The eigenvalues $d_{i}$ and the eigenvectors of the $S$ matrix constitute the diagonal matrix $D$ and the unitary matrix $U=\left(u_{i j}\right)$ respectively; columns of the unitary $U$ operator are the normalized eigenvectors of the $S$ matrix. Then the Kraus operators:

$$
E_{i}=\sum_{j} \sqrt{d_{i}} u_{j i} \mathcal{G}_{j}
$$

yield the Kraus decomposition of the dynamical map $\phi_{t}$ :

$$
\phi_{t}\left(\hat{\rho}_{S}(0)\right)=\sum_{k} \hat{E}_{k}(t) \hat{\rho}_{S}(0) \hat{E}_{k}^{\dagger}(t)
$$

Therefore, the chain of the construction is established: from a master equation to calculate $L$, then via relation (7) to obtain the matrix $F$ and, due to eq.(8) and diagonalization eq.(10) of the Choi matrix to calculate the Kraus operators eq.(11).

\section{THE GENERALIZED AMPLITUDE DAMPING CHANNEL}

The standard master equation for the amplitude damping process, at absolute zero, $T=0 \mathrm{~K}$, reads $[2]$ :

$$
\frac{d \hat{\rho}_{S}(t)}{d t}=\frac{\gamma}{2}\left(2 \hat{\sigma}_{-} \hat{\rho}_{S}(t) \hat{\sigma}_{+}-\hat{\sigma}_{+} \hat{\sigma}_{-} \hat{\rho}_{S}(t)-\hat{\rho}_{S}(t) \hat{\sigma}_{+} \hat{\sigma}_{-}\right)
$$

while the corresponding standard Kraus operators:

$$
\hat{E}_{0}=|0\rangle\langle 0|+\sqrt{1-\lambda(t)}| 1\rangle\left\langle 1\left|, \quad \hat{E}_{1}=\sqrt{\lambda(t)}\right| 0\right\rangle\langle 1|
$$

and the Pauli operators $\hat{\sigma}_{z}=|0\rangle\langle 0|-| 1\rangle\left\langle 1\left|, \hat{\sigma}_{x}=\right| 0\right\rangle\langle 1|+| 1\rangle\left\langle 0\left|, \hat{\sigma}_{y}=\imath\right| 0\right\rangle\langle 1|-\imath| 1\rangle\langle 0|$, and $\hat{\sigma}_{ \pm}=\frac{1}{2}\left(\hat{\sigma}_{x} \pm \imath \hat{\sigma}_{y}\right)$. 
To describe the amplitude damping process for all temperatures, the following Hamiltonian is often regarded [11]:

$$
\hat{H}=\frac{\omega_{0}}{2} \hat{\sigma}_{z}+\int_{0}^{\omega_{\max }} d \omega \hat{a}_{\omega}^{\dagger} \hat{a}_{\omega}+\int_{0}^{\omega_{\max }} d \omega h(\omega)\left(\hat{a}_{\omega}^{\dagger} \hat{\sigma}_{-}+\hat{a}_{\omega} \hat{\sigma}_{+}\right)
$$

The first term on the right side of eq.(15) denotes the system's self-Hamiltonian, the second denotes the self-Hamiltonian of the environment (a thermal bath of linear noninteracting harmonic oscillators and $\hat{a}_{\omega}$ representing the bosonic "annihilation" operator for the frequency $\omega$ ) while the last term represents the interaction with the couplingcoefficients $h(\omega)$. $\omega_{\max }$ is the 'cutoff frequency' for the bath's oscillators; one may take the limit $\omega_{\max } \rightarrow \infty$ providing that the $h(\omega)$ sufficiently quickly decreases.

As distinct from eq.(13), the microscopic Markovian master equation, in interaction picture, derived from Hamiltonian (15) reads [11]:

$$
\begin{aligned}
\frac{d \hat{\rho}_{S}(t)}{d t} & =-i\left[\left(\frac{\Delta}{2}+\Delta^{\prime}\right) \hat{\sigma}_{z}, \hat{\rho}_{S}(t)\right] \\
& +2 \pi J\left(\omega_{0}\right)\left(\left\langle n\left(\omega_{0}\right)\right\rangle+1\right)\left[\hat{\sigma}_{-} \hat{\rho}_{S}(t) \hat{\sigma}_{+}-\frac{1}{2}\left\{\hat{\sigma}_{+} \hat{\sigma}_{-}, \hat{\rho}_{S}(t)\right\}\right] \\
& +2 \pi J\left(\omega_{0}\right)\left\langle n\left(\omega_{0}\right)\right\rangle\left[\hat{\sigma}_{+} \hat{\rho}_{S}(t) \hat{\sigma}_{-}-\frac{1}{2}\left\{\hat{\sigma}_{-} \hat{\sigma}_{+}, \hat{\rho}_{S}(t)\right\}\right],
\end{aligned}
$$

where $\Delta=\mathrm{P} . \mathrm{V} \cdot \int_{0}^{\omega_{\max }} d \omega^{\prime} \frac{J\left(\omega^{\prime}\right)}{\omega_{0}-\omega^{\prime}}$ and $\Delta^{\prime}=\mathrm{P} . \mathrm{V} \cdot \int_{0}^{\omega_{\max }} d \omega^{\prime} \frac{J\left(\omega^{\prime}\right)\left\langle n\left(\omega^{\prime}\right)\right\rangle}{\omega_{0}-\omega^{\prime}}$ denote the Lamb-like shift and the Stark-like shift contributions from the vacuum and the thermal field, respectively, and curly brackets stand for anti-commutator. P.V. stands for the Cauchy principal value of the integral. $J(\omega)$ represents the spectral density of the bath.

The master equation (16) reduces to the standard $\mathrm{AD}$ master equation (13) for $T=0 \mathrm{~K}$, and is therefore often called generalized amplitude damping (GAD) channel. Below, due to the procedure described in Section 2, from eq.(16) we derive the GAD Kraus operators, which will turn out to be unitary equivalent with the known GAD Kraus operators [12]:

$$
\begin{array}{ll}
E_{0} \equiv \sqrt{p}\left[\begin{array}{cc}
\sqrt{1-\lambda(t)} & 0 \\
0 & 1
\end{array}\right] ; & E_{1} \equiv \sqrt{p}\left[\begin{array}{ll}
0 & 0 \\
\sqrt{\lambda(t)} & 0
\end{array}\right] ; \\
E_{2} \equiv \sqrt{1-p}\left[\begin{array}{cc}
1 & 0 \\
0 & \sqrt{1-\lambda(t)}
\end{array}\right] ; & E_{3} \equiv \sqrt{1-p}\left[\begin{array}{ll}
0 & \sqrt{\lambda(t)} \\
0 & 0
\end{array}\right],
\end{array}
$$

where $\lambda(t) \equiv 1-e^{-\gamma_{0}\left(2 N_{\mathrm{th}}+1\right) t} ; p \equiv \frac{N_{\mathrm{th}}+1}{2 N_{\mathrm{th}}+1} . N_{\mathrm{th}}=\left[e^{(\omega / T)}-1\right]^{-1}$ while $\gamma$ appears in $(13)$.

To ease the calculation, we introduce the following notation: $\mathbf{x}=\frac{\Delta}{2}+\Delta^{\prime}, \mathbf{y}=$ $2 \pi J\left(\omega_{0}\right)\left(\left\langle n\left(\omega_{0}\right)\right\rangle+1\right)>\mathbf{z}=2 \pi J\left(\omega_{0}\right)\left\langle n\left(\omega_{0}\right)\right\rangle \geq 0$ with which the equation (16) reads:

$$
\begin{aligned}
\frac{d \hat{\rho}_{S}(t)}{d t} & =-i \mathbf{x}\left[\hat{\sigma}_{z}, \hat{\rho}_{S}(t)\right] \\
& +\mathbf{y}\left[\hat{\sigma}_{-} \hat{\rho}_{S}(t) \hat{\sigma}_{+}-\frac{1}{2}\left\{\hat{\sigma}_{+} \hat{\sigma}_{-}, \hat{\rho}_{S}(t)\right\}\right] \\
& +\mathbf{z}\left[\hat{\sigma}_{+} \hat{\rho}_{S}(t) \hat{\sigma}_{-}-\frac{1}{2}\left\{\hat{\sigma}_{-} \hat{\sigma}_{+}, \hat{\rho}_{S}(t)\right\}\right] .
\end{aligned}
$$


Now, from (18) and using eq.(6) from the body text, the $\hat{\sigma}_{z}$-representation of the $L$ matrix takes the form:

$$
L=\left(\begin{array}{cccc}
0 & 0 & 0 & 0 \\
0 & \frac{1}{2}(-\mathbf{y}-\mathbf{z}) & -2 \mathbf{x} & 0 \\
0 & 2 \mathbf{x} & \frac{1}{2}(-\mathbf{y}-\mathbf{z}) & 0 \\
\mathbf{z}-\mathbf{y} & 0 & 0 & -\mathbf{y}-\mathbf{z}
\end{array}\right)
$$

In order to facilitate the calculation of the exponential $F$ matrix, we multiply the $L$ matrix by $\frac{2}{(\mathbf{y}+\mathbf{z})}$ that allows introduction of new variables: $\theta=\frac{4 \mathbf{x}}{\mathbf{y}+\mathbf{z}}, \Omega=-\frac{2(\mathbf{y}-\mathbf{z})}{(\mathbf{y}+\mathbf{z})}$, $\tau=\frac{(\mathbf{y}+\mathbf{z})}{2} t ; \Omega \in[-2,0), \tau \in(-\infty, \infty)$. Then follows:

$$
\frac{2 L}{\mathbf{y}+\mathbf{z}}=\left(\begin{array}{cccc}
0 & 0 & 0 & 0 \\
0 & -1 & -\theta & 0 \\
0 & \theta & -1 & 0 \\
\Omega & 0 & 0 & -2
\end{array}\right)
$$

and

$$
F=e^{\frac{2 L}{\mathbf{y}+\mathbf{z}} \tau}
$$

which obtains the form:

$$
F=\left(\begin{array}{cccc}
1 & 0 & 0 & 0 \\
0 & e^{-\tau} \cos (\theta \tau) & -e^{-\tau} \sin (\theta \tau) & 0 \\
0 & e^{-\tau} \sin (\theta \tau) & e^{-\tau} \cos (\theta \tau) & 0 \\
e^{-\tau} \Omega \sinh (\tau) & 0 & 0 & e^{-2 \tau}
\end{array}\right)
$$

From eq.(22) we obtain the corresponding Choi matrix (Section 2), whose diagonalization gives the following set of eigenvalues:

$$
\begin{array}{r}
-\frac{1}{4} e^{-2 \tau}\left(-1+e^{2 \tau}\right)(-2+\Omega), \\
\frac{1}{4} e^{-2 \tau}\left(-1+e^{2 \tau}\right)(2+\Omega), \\
\frac{1}{4} e^{-2 \tau}\left(2+2 e^{2 \tau}-\sqrt{16 e^{2 \tau}+\Omega^{2}-2 e^{2 \tau} \Omega^{2}+e^{4 \tau} \Omega^{2}}\right), \\
\frac{1}{4} e^{-2 \tau}\left(2+2 e^{2 \tau}+\sqrt{16 e^{2 \tau}+\Omega^{2}-2 e^{2 \tau} \Omega^{2}+e^{4 \tau} \Omega^{2}}\right),
\end{array}
$$

and the respective non-normalized eigenvectors: 


$$
\begin{array}{r}
\left\{0, \frac{1}{2} i e^{-\tau}\left(-1+e^{2 \tau}\right) \operatorname{Csch}[\tau], 1,0\right\}, \\
\left\{0,-\frac{1}{2} i e^{-\tau}\left(-1+e^{2 \tau}\right) \operatorname{Csch}[\tau], 1,0\right\}, \\
\left\{\frac{e^{-\tau}\left(-\sqrt{16 e^{2 \tau}+\Omega^{2}-2 e^{2 \tau} \Omega^{2}+e^{4 \tau} \Omega^{2}}+4 e^{\tau} \operatorname{Cos}[\theta \tau]\right)}{2(-2 i \operatorname{Sin}[\theta \tau]+\Omega \operatorname{Sinh}[\tau])}, 0,0,1\right\}, \\
\left\{\frac{e^{-\tau}\left(\sqrt{16 e^{2 \tau}+\Omega^{2}-2 e^{2 \tau} \Omega^{2}+e^{4 \tau} \Omega^{2}}+4 e^{\tau} \operatorname{Cos}[\theta \tau]\right)}{2(-2 i \operatorname{Sin}[\theta \tau]+\Omega \operatorname{Sinh}[\tau])}, 0,0,1\right\} .
\end{array}
$$

Hence we obtain the first two Kraus matrices for GAD, eq.(4):

$$
\begin{gathered}
\mathbb{E}_{1}=\left(\begin{array}{cc}
0 & 0 \\
\frac{1}{2} i \sqrt{\left(e^{-2 \tau}-1\right)(\Omega-2)} & 0
\end{array}\right), \\
\mathbb{E}_{2}=\left(\begin{array}{cc}
0 & -\frac{1}{2} i \sqrt{\left(1-e^{-2 \tau}\right)(\Omega+2)} \\
0 & 0
\end{array}\right) .
\end{gathered}
$$

By introducing:

$$
\begin{gathered}
A=-2 i \sin [\theta \tau]+\Omega \sinh [\tau], \\
B_{ \pm}=e^{-4 \tau}\left(2+2 e^{2 \tau} \pm \sqrt{\Omega^{2}+e^{2 \tau}\left(16+\left(-2+e^{2 \tau}\right) \Omega^{2}\right)}\right) \\
C_{ \pm}=e^{-2 \tau}\left(\sqrt{\Omega^{2}+e^{2 \tau}\left(16+\left(-2+e^{2 \tau}\right) \Omega^{2}\right)} \pm 4 e^{\tau} \cos [\theta \tau]\right)^{2} \\
D=4 e^{\tau-i \theta \tau}
\end{gathered}
$$

and

$$
E_{ \pm}=\left(1-e^{2 \tau}\right) \Omega \pm \sqrt{\Omega^{2}+e^{2 \tau}\left(16+\left(-2+e^{2 \tau}\right) \Omega^{2}\right)},
$$

another pair of Kraus matrices, $\mathbb{E}_{3}$ and $\mathbb{E}_{4}$, can be written as:

$$
\mathbb{E}_{3}=\frac{\sqrt{\frac{|A|^{2} B_{-}}{4|A|^{2}+C_{-}}}}{2 \sqrt{2} A}\left(\begin{array}{cc}
D-E_{+} & 0 \\
0 & D^{*}+E_{-}
\end{array}\right)
$$

and

$$
\mathbb{E}_{4}=\frac{\sqrt{\frac{|A|^{2} B_{+}}{4|A|^{2}+C_{+}}}}{2 \sqrt{2} A}\left(\begin{array}{cc}
D-E_{-} & 0 \\
0 & D^{*}+E_{+}
\end{array}\right)
$$

It is straightforward yet tedious task to confirm the completeness relation $\sum_{k} \hat{E}_{k}(t)^{\dagger} \hat{E}_{k}(t)=$ $\hat{I}$ ( $\hat{I}$ is the identity operator) for the Kraus matrices eqs. (25), (26), (32) and (33). 
For the bath on $T=0 K$, the parameters $\mathbf{x}=0=\mathbf{y}$, equivalently $\theta=0, \Omega=-2$, the GAD master equation eq.(16) reduces to the standard AD master equation eq.(13). Now placing $\theta=0, \Omega=-2$ in eqs.(25)-(33), we obtain:

$$
\begin{gathered}
\mathbb{E}_{1}=\left(\begin{array}{cc}
0 & 0 \\
i \sqrt{1-e^{-2 \tau}} & 0
\end{array}\right), \\
\mathbb{E}_{2}=0 \\
\mathbb{E}_{3}=0
\end{gathered}
$$

and

$$
\mathbb{E}_{4}=\left(\begin{array}{cc}
-e^{-\tau} & 0 \\
0 & -1
\end{array}\right)
$$

It is easy to prove the completeness relation for the Kraus matrices eqs.(34) and (37).

The matrices $\mathbb{E}_{1}^{\prime}=-i \mathbb{E}_{1}$ and $\mathbb{E}_{4}^{\prime}=-\mathbb{E}_{4}$ are unitary-equivalent with the standard $\mathrm{AD}$ Kraus operators eq.(14). That is, the sets eqs.(34)-(37) and eq.(14) describe the same process.

Unitary equivalence of the Kraus matrices (25)-(33) and the GAD Kraus matrices (17) follows from the following observations. First, the GAD Kraus matrices eq.(5) reduce to standard ones eq.(14) for $N_{\text {th }}=0$ i.e. $p=1$ (in our notation these are: $\theta=0, \Omega=-2$ ). Second, for both sets of the Kraus operators, (25)-(33), and eq.(17), it easily follows:

$$
\begin{aligned}
\phi_{\tau}(\hat{I}) & =\hat{I}+\frac{\Omega}{2}\left(1-e^{-2 \tau}\right) \hat{\sigma}_{z}, \\
\phi_{\tau}\left(\hat{\sigma}_{x}\right) & =e^{-\tau}\left(\hat{\sigma}_{x} \cos \theta \tau+\hat{\sigma}_{y} \sin \theta \tau\right), \\
\phi_{\tau}\left(\hat{\sigma}_{y}\right) & =e^{-\tau}\left(\hat{\sigma}_{y} \cos \theta \tau-\hat{\sigma}_{x} \sin \theta \tau\right), \\
\phi_{\tau}\left(\hat{\sigma}_{z}\right) & =e^{-2 \tau} \hat{\sigma}_{z},
\end{aligned}
$$

which, bearing in mind $\hat{\rho}=\frac{1}{2}(\hat{I}+\vec{n} \cdot \hat{\vec{\sigma}})$, gives rise to:

$$
\begin{aligned}
\phi_{\tau}(\hat{\rho}) & =\frac{1}{2}\left[\hat{I}+e^{-\tau} \sin v \cos (u+\theta \tau) \hat{\sigma}_{x}+e^{-\tau} \sin v \sin (u+\theta \tau) \hat{\sigma}_{y}\right. \\
& \left.+\left(\frac{\Omega}{2}\left(1-e^{-2 \tau}\right)+e^{-2 \tau} \cos v\right) \hat{\sigma}_{z}\right]
\end{aligned}
$$

i.e. to:

$$
\begin{aligned}
\phi_{t}(\hat{\rho}) & =\frac{1}{2}\left[\hat{I}+e^{-\frac{1}{2} t(\mathbf{y}+\mathbf{z})} \cos (u+2 t \mathbf{x}) \sin v \hat{\sigma}_{x}+e^{-\frac{1}{2} t(\mathbf{y}+\mathbf{z})} \sin v \sin (u+2 t \mathbf{x}) \hat{\sigma}_{y}\right. \\
& \left.+\frac{\left(\left(-1+2 e^{-t(\mathbf{y}+\mathbf{z})}\right) \mathbf{y}+\mathbf{z}\right) \cos v}{\mathbf{y}+\mathbf{z}} \hat{\sigma}_{z}\right] .
\end{aligned}
$$

Expressions eq.(39) and eq.(40) are solutions of the master equation eq.(16). Unitaryequivalent Kraus matrices eqs.(25)-(33) and eq.(17) describe the same process.

For completeness, with the use of eqs.(38), below we compare the temporal behaviors of the Bloch sphere for the standard $\mathrm{AD}$ and $\mathrm{GAD}$ channels. Also we study the GAD 


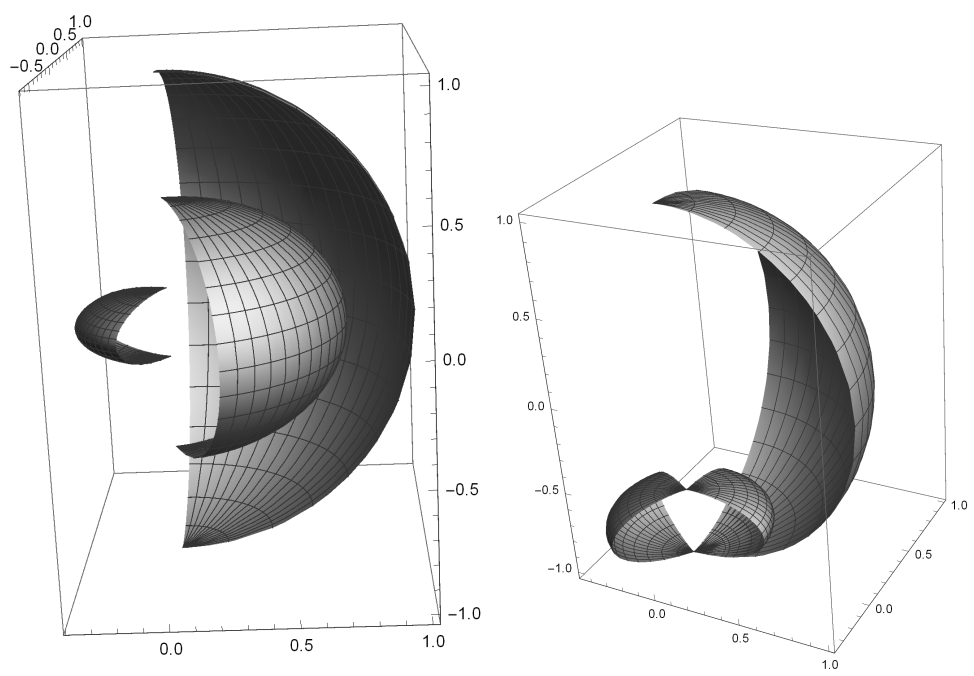

Figure 1: (Left) Representation of the GAD process for high temperature: the big sphere is for the initial instant of time $t=0$-the unchanged Bloch sphere. For $t=0.05$, both smaller and larger ellipsoids pertain to the GAD channel for $T=300$ and $T=100$, respectively. (Right) A representation for low temperature $(T=1)$ : the big sphere is for the initial instant of time $t=0$. For $t=2.5$, there is only one ellipsoid for both, the standard $\mathrm{AD}$ and the GAD channel, exhibiting their match in this temperature regime. The parameters: $\alpha=0.02, \omega_{0}=10$ and $\omega_{c}=15$.

channel for various (high) temperatures via investigating temporal behavior of the Bloch sphere volume.

Fig.1(right) exhibits unchangeability of the "ground" state $|1\rangle$ on $T=0 \mathrm{~K}$ while Fig.1(left) shows instability (finite probability for excitation) of the ground state for the finite temperature range; of course, this physical observation is well known from the application of the quantum-optical master equation to a two-state atom [13]. On the other hand, Fig.1(left) reveals a faster change of the "excited" state $|0\rangle$ for higher temperatures.

Time dependence of the Bloch-sphere volume is:

$$
V(\tau)=\frac{4 \pi}{3} e^{-4 \tau}
$$

equivalently $V(t)=\frac{4 \pi}{3} e^{-2(\mathbf{z}+\mathbf{y}) t}$. The relative change of the Bloch sphere volume, $\kappa(t)=$ $\frac{1}{V_{0}} \frac{d V(t)}{d t}$ :

$$
\kappa(t)=-2(\mathbf{z}+\mathbf{y}) e^{-2(\mathbf{z}+\mathbf{y}) t} .
$$

Eq.(42) regarding GAD process is presented in Fig.2;

Finally, GAD Kraus operators, eqs.(25)-(33) and eq.(17), can be shown to take the following forms in the asymptotic limit , $\tau \rightarrow \infty$ :

$$
\mathbb{E}_{1}^{\mathrm{Asym}}=\left(\begin{array}{cc}
0 & 0 \\
\frac{i \sqrt{2-\Omega}}{2} & 0
\end{array}\right)
$$




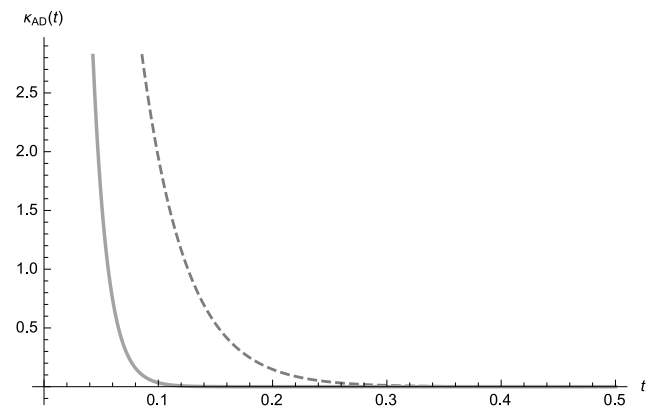

Figure 2: The relative change of the Bloch sphere volume for the high-temperature GAD process. The dashed line is for $T=100$ and the thick one for $T=300$. The parameters: $\alpha=0.02, \omega_{0}=10$ and $\omega_{c}=15$.

$$
\begin{aligned}
& \mathbb{E}_{2}^{\text {Asym }}=\left(\begin{array}{cc}
0 & -\frac{i \sqrt{\Omega+2}}{2} \\
0 & 0
\end{array}\right) \\
& \mathbb{E}_{3}^{\text {Asym }}=\left(\begin{array}{cc}
0 & 0 \\
0 & -\frac{\sqrt{2-\Omega}}{2}
\end{array}\right) \\
& \mathbb{E}_{4}^{\text {Asym }}=\left(\begin{array}{cc}
\frac{\sqrt{\Omega+2}}{2} & 0 \\
0 & 0
\end{array}\right)
\end{aligned}
$$

from which it is clear that, asymptotically, the actions of the standard AD channel, eqs.((34)-(37)), and of the GAD channel, eqs.((43)-(46)) , are not mutually equivalent, except for the choice $\Omega=-2$ for the GAD channel.

\section{THE PHASE DAMPING CHANNEL}

The phase damping (PD) quantum channel models pure decoherence without loss of energy for a single-qubit system. The Hamiltonian for the total (closed) system is given by [11]:

$$
\hat{H}=\frac{\omega_{0}}{2} \hat{\sigma}_{z}+\int_{0}^{\omega_{\max }} d \omega \hat{a}_{\omega}^{\dagger} \hat{a}_{\omega}+\hat{\sigma}_{z} \otimes \int_{0}^{\omega_{\max }} d \omega h(\omega)\left(\hat{a}_{\omega}^{\dagger}+\hat{a}_{\omega}\right)
$$

Notation and the meaning of the terms in eq.(47) are the same as in eq.(15).

The model eq.(47) gives rise to the following microscopic Markovian master equation in the interaction picture [11]:

$$
\frac{d \hat{\rho}_{S}(t)}{d t}=r\left(\hat{\sigma}_{z} \hat{\rho}_{S}(t) \hat{\sigma}_{z}-\hat{\rho}_{S}(t)\right)
$$

whereby the decay rate $r$ [11]:

$$
r=2 \pi \lim _{\omega \rightarrow 0} J(|\omega|)\langle n(|\omega|)\rangle
$$


under assumption $\lim _{\omega \rightarrow 0} J(|\omega|)=0 . J(\omega)$ is the spectral density of the bath while $\langle n(\omega)\rangle$ is the mean number of the bosons for the thermal state of the bath with the frequency $\omega$.

Following the recipe of Section 2:

$$
\begin{gathered}
L=\left(\begin{array}{cccc}
0 & 0 & 0 & 0 \\
0 & -2 r & 0 & 0 \\
0 & 0 & -2 r & 0 \\
0 & 0 & 0 & 0
\end{array}\right), \\
F=\left(\begin{array}{cccc}
1 & 0 & 0 & 0 \\
0 & e^{-2 r t} & 0 & 0 \\
0 & 0 & e^{-2 r t} & 0 \\
0 & 0 & 0 & 1
\end{array}\right), \\
S=\left(\begin{array}{cccc}
1+e^{-2 r t} & 0 & 0 & 0 \\
0 & 0 & 0 & 0 \\
0 & 0 & 0 & 0 \\
0 & 0 & 0 & 1-e^{-2 r t}
\end{array}\right),
\end{gathered}
$$

which give rise to the following Kraus operators:

$$
\begin{aligned}
& \mathbb{E}_{1}=\left(\begin{array}{cc}
\frac{\sqrt{1-e^{-2 r t}}}{\sqrt{2}} & 0 \\
0 & -\frac{\sqrt{1-e^{-2 r t}}}{\sqrt{2}}
\end{array}\right), \\
& \mathbb{E}_{2}=\left(\begin{array}{cc}
\frac{\sqrt{1+e^{-2 r t}}}{\sqrt{2}} & 0 \\
0 & \frac{\sqrt{1+e^{-2 r t}}}{\sqrt{2}}
\end{array}\right) .
\end{aligned}
$$

These matrices are the $\hat{\sigma}_{z}=|0\rangle\langle 0|-| 1\rangle\langle 1|$ representations of the well known Kraus operators for the PD channel [2]:

$$
\hat{E}_{0}=\sqrt{1-\frac{p(t)}{2}} \hat{I}, \quad \hat{E}_{1}=\sqrt{\frac{p(t)}{2}} \hat{\sigma}_{z}
$$

where $p(t) \equiv 1-e^{-2 r t}$ while the completeness relation $\sum_{k} \hat{E}_{k}(t)^{\dagger} \hat{E}_{k}(t)=\hat{I}$ is satisfied.

From Kraus operators, eqs.(53) and (54) easily follows

$$
\begin{aligned}
\phi_{\tau}(\hat{I}) & =\hat{I}, \\
\phi_{\tau}\left(\hat{\sigma}_{x}\right) & =e^{-2 r t} \hat{\sigma}_{x}, \\
\phi_{\tau}\left(\hat{\sigma}_{y}\right) & =e^{-2 r t} \hat{\sigma}_{y}, \\
\phi_{\tau}\left(\hat{\sigma}_{z}\right) & =\hat{\sigma}_{z} .
\end{aligned}
$$

Hence the solution of eq.(48):

$$
\begin{aligned}
\phi_{\tau}(\hat{\rho}) & =\frac{1}{2}\left[\hat{I}+e^{-2 r t} \sin v \cos u \hat{\sigma}_{x}+e^{-2 r t} \sin v \sin u \hat{\sigma}_{y}\right. \\
& \left.+\cos v \hat{\sigma}_{z}\right]
\end{aligned}
$$


for the initial state $\hat{\rho}=\frac{1}{2}(\hat{I}+\vec{n} \cdot \hat{\vec{\sigma}}) ; n=\left(n_{x}, n_{y}, n_{z}\right)$. Notice diagonalizability of the state eq.(57) for long times $(t \rightarrow \infty)$ in the $\hat{\sigma}_{z}$ eigenbasis, which becomes the "pointer basis" for the decoherence process [1] induced by the environment.

\section{CONCLUSION}

Detailed microscopic analysis of the differential form of the amplitude damping and phase damping processes on a single qubit gives rise to the Kraus operators that describe exactly the same process as the standard Kraus operators widely used for these processess.

\section{REFERENCES}

[1] M. A. Nielsen, I. L. Chuang, Quantum Computation and Quantum Information Cambridge Univ. Press, Cambridge, (2000).

[2] K. M. Fonseca Romero and R. Lo Franco, Simple Non-Markovian Microscopic Models for the Depolarizing Channel of a Single Qubit, Physica Scripta 86, 065004 (2012).

[3] Principles of quantum computation and information Volume 2, Benenti G., Casati G., Strini G., London (2007).

[4] Q.G. Chen, D.A. Church, B.-G. Englert, C. Henkel, B. Rohwedder, M.O. Scully, M.S. Zubairy, Quantum Computing Devices: Principles, Designs, and Analysis, Chapman and Hall (2006).

[5] B. Bylicka, D. Chruściński, S. Maniscalco, Non-Markovianity and reservoir memory of quantum channels: a quantum information theory perspective. Sci. Rep. 4, 5720 (2014).

[6] D. C. Marinescu and G. M. Marinescu, Classical and Quantum Information Elsevier, Amsterdam, (2012).

[7] A.B. Klimov and L.L. Sanchez-Soto, Depolarization for quantum channels with higher symmetries, Physica Scripta T140, 014009 (2010).

[8] E. Andersson, J. D. Cresser, and M. J. W. Hall, J. Mod. Opt. 54, 1695 (2007). 
[9] M. Arsenijević , J. Jeknic-Dugić , M. Dugić: Generalized Kraus operators for the one-qubit depolarizing quantum channel, ArXiv: 1512.07843v2.

[10] M.-D. Choi, Lin. Alg. Appl. 10285 (1975).

[11] A. Rivas, S.F. Huelga: Open Quantum Systems - An Introduction, Springer Briefs in Physics (2012).

[12] R. Srikanth, , S. Banerjee: Squeezed generalized amplitude damping channel, Phys. Rev. A77, 012318 (2008).

[13] H.P. Breuer, F. Petruccione: The Theory of Open Quantum Systems, Clarendon Press, Oxford (2002). 